\title{
Comment on "Ultrafast Demagnetization Measurements Using Extreme Ultraviolet Light: Comparison of Electronic and Magnetic Contributions"
}

\author{
Boris Vodungbo, ${ }^{*}$ Julien Gautier, Guillaume Lambert, and Philippe Zeitoun \\ Laboratoire d'Optique Appliquée, ENSTA ParisTech-CNRS UMR 7639-École Polytechnique, \\ Chemin de la Hunière, 91761 Palaiseau, France \\ Jan Lüning \\ Laboratoire de Chimie Physique-Matière et Rayonnement, Université Pierre et Marie Curie-CNRS UMR 7614, \\ 11 rue Pierre et Marie Curie, 75005 Paris, France \\ (Received 10 January 2013; revised manuscript received 19 March 2013; published 4 September 2013)
}

\begin{abstract}
The time-resolved transverse magneto-optical Kerr effect using high-order harmonics is a very promising technique to study ultrafast magnetization dynamics. Recently, it was claimed that this technique was free of any nonmagnetic artifact. Here, we show that this report was not fully supported. We propose simple additional measurements that can unambiguously demonstrate this affirmation.
\end{abstract}

DOI: 10.1103/PhysRevX.3.038001

In a recent article, La-O-Vorakiat and co-workers [1] report data on ultrafast demagnetization dynamics obtained using an extreme ultraviolet femtosecond light source based on high-order harmonics generation. This type of femtosecond light source has recently been shown to be ideally suited for studying ultrafast magnetization dynamics, since it offers in comparison to conventional femtosecond infrared lasers the following key advantages: enhanced magnetic sensitivity, enhanced time resolution, element specificity, and nanometer spatial resolution [2-5]. Furthermore, it has also been shown recently that circularly polarized harmonics could be obtained [6], opening the way to the study of more complex magnetic heterostructures. In their study, the authors probed the sample's magnetization with harmonics resonant to the $\mathrm{Ni}$ and $\mathrm{Fe} M_{2,3}$ absorption edges that exhibit a very strong transverse magneto-optical Kerr effect. While we agree with the authors that using high harmonics is a promising experimental approach for studying ultrafast magnetization dynamics, we have to note that one of their main conclusions, namely, that the $M_{2,3}$-edge transverse magneto-optical Kerr effect signal in this time-resolved experiment is essentially free of transient artifacts that are not magnetic in origin, is based on an incorrect chain of arguments.

The error is in the estimation of what the maximum contribution of nonmagnetic artifacts to the observed variation of the asymmetry ratio may be. The estimate of this contribution to the experiment realized in $p$ geometry is based on the observation that in $s$ geometry (no magnetic contribution in this geometry), only a small variation is

\footnotetext{
*boris.vodungbo@ensta-paristech.fr

Published by the American Physical Society under the terms of the Creative Commons Attribution 3.0 License. Further distribution of this work must maintain attribution to the author(s) and the published article's title, journal citation, and DOI.
}

Subject Areas: Magnetism, Optics

observed in the time-resolved reflectivity measurement. The authors argue that in the worst case, this variation, due to a modification of the optical index $n=1-\delta+\imath \beta$, is caused either by a variation of $\delta$ or $\beta$ and that consequently any such variation has to be small. From this remark, the authors then conclude that any nonmagnetic contribution to the asymmetry variation observed in $p$ geometry has to be small, too. However, the presented argument is incorrect, since weak variations of the $s$ reflectivity $R_{s}$ may originate from large simultaneous variations of $\delta$ and $\beta$. We exhibit this possibility in Figs. 1(a) and 1(b) using the optical index and magneto-optical constant of $\mathrm{Ni}$ at $67.7 \mathrm{eV}$ from Refs. [7,8].

We also remark that it is not indicated which line of Eq. (3) in their manuscript is used for the asymmetry calculations. However, this precision is necessary, since the third line of Eq. (3) cannot be derived by a proper expansion of line 2 and is not defined for the experimentally employed incidence angle of $45^{\circ}$. Also, it has to be noted that expression 1 is valid only for a semi-infinite magnetic plane. In the case of a complex system consisting of one or several thin layers, the correct expression is more complex [9], and, as demonstrated before [10,11], this complexity can lead to unexpected effects that are not discussed by the authors. Furthermore, we remark that the photon energy used for magnetic measurements in $p$ geometry, $66.2 \mathrm{eV}$, is not the same as the one used for reflectivity measurements in $s$ geometry, $67.7 \mathrm{eV}$. This renders these two sets of data difficult to compare, since reflectivity at an absorption resonance is highly dependent on the photon energy [12]. Finally, the magneto-optical constant $\epsilon_{x y}$, which is needed for the calculation of the asymmetry, is not given, nor is its origin specified by the authors.

We conclude that for the above reasons, the given argumentation is insufficient to support the presented conclusion. However, we would like to point out that the 

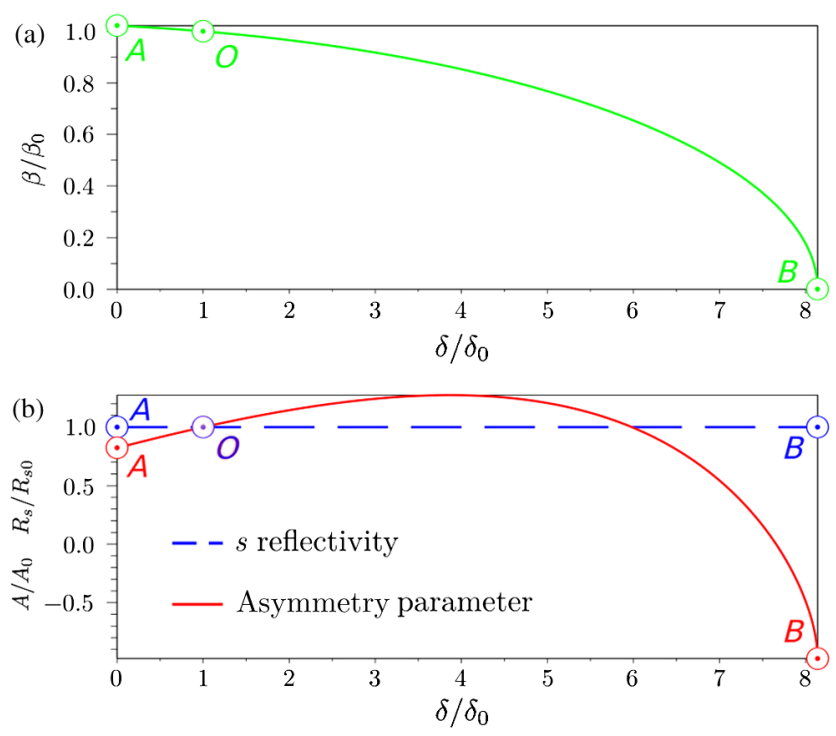

FIG. 1. (a) Trajectory in the optical index space for $R_{s}(\delta, \beta)=$ $R_{s 0}, R_{s 0}$ being the ground-state $s$ reflectivity of nickel at $67.7 \mathrm{eV}$ using $n_{0}=1-\delta_{0}+\imath \beta_{0}\left(\delta_{0}=0.01066, \beta_{0}=0.10030\right)$. One notes that very strong variations of the optical index $n$ can result in very small variations of $R_{s}$. (b) Normalized asymmetry parameter (to $A_{0}$, asymmetry for $n_{0}$ ) and normalized $s$ reflectivity (to $R_{s 0}, s$ reflectivity for $n_{0}$ ) along the same optical index trajectory (from points $A$ to $B$, point $O$ represents the value for $n_{0}$ ). Although $R_{s}$ remains constant, $A$ presents strong variations and even changes its sign. Asymmetry has been calculated using Eq. (3) of Ref. [1] (with no approximation) and $\epsilon_{x y}=-0.0051-\imath 0.0050$.

possibility of a variation of $n$ along an iso- $s$ reflectivity trajectory in the $\delta$ - $\beta$ space could be eliminated experimentally, e.g., by measuring the $s$ reflectivity for a series of different incident angles. This experiment should be straightforward to do.

[1] C. La-O-Vorakiat, E. Turgut, C. A. Teale, H. C. Kapteyn, M. M. Murnane, S. Mathias, M. Aeschlimann, C. M. Schneider, J. M. Shaw, H.T. Nembach, and T. J. Silva, Ultrafast Demagnetization Measurements Using Extreme Ultraviolet Light: Comparison of Electronic and Magnetic Contributions, Phys. Rev. X 2, 011005 (2012).

[2] C. La-O-Vorakiat, M. Siemens, M. M. Murnane, H. C. Kapteyn, S. Mathias, M. Aeschlimann, P. Grychtol, R. Adam, C. M. Schneider, J. M. Shaw, H. Nembach, and T. J. Silva, Ultrafast Demagnetization Dynamics at the $M$ Edges of Magnetic Elements Observed Using a Tabletop
High-Harmonic Soft X-Ray Source, Phys. Rev. Lett. 103, 257402 (2009).

[3] B. Vodungbo, A. B. Sardinha, J. Gautier, G. Lambert, M. Lozano, S. Sebban, E. Meltchakov, F. Delmotte, V. LopezFlores, J. Arabski, C. Boeglin, E. Beaurepaire, R. Delaunay, J. Lüning, and P. Zeitoun, Table-Top Resonant Magnetic Scattering with Extreme Ultraviolet Light from High-Order Harmonic Generation, Europhys. Lett. 94, 54003 (2011).

[4] S. Mathias, C. La-O-Vorakiat, P. Grychtol, P. Granitzka, E. Turgut, J. M. Shaw, R. Adam, H. T. Nembach, M.E. Siemens, S. Eich, C.M. Schneider, T. J. Silva, M. Aeschlimann, M.M. Murnane, and H.C. Kapteyn, Probing the Timescale of the Exchange Interaction in a Ferromagnetic Alloy, Proc. Natl. Acad. Sci. U.S.A. 109, 4792 (2012).

[5] B. Vodungbo, J. Gautier, G. Lambert, A. B. Sardinha, M. Lozano, S. Sebban, M. Ducousso, W. Boutu, K. Li, B. Tudu, M. Tortarolo, R. Hawaldar, R. Delaunay, V. LópezFlores, J. Arabski, C. Boeglin, H. Merdji, P. Zeitoun, and J. Lüning, Laser-Induced Ultrafast Demagnetization in the Presence of a Nanoscale Magnetic Domain Network, Nat. Commun. 3, 999 (2012).

[6] B. Vodungbo, A. B. Sardinha, J. Gautier, G. Lambert, C. Valentin, M. Lozano, G. Iaquaniello, F. Delmotte, S. Sebban, J. Lüning, and P. Zeitoun, Polarization Control of High Order Harmonics in the EUV Photon Energy Range, Opt. Express 19, 4346 (2011).

[7] B.L. Henke, E. M. Gullikson, and J.C. Davis, X-Ray Interactions: Photoabsorption, Scattering, Transmission, and Reflection at $e=50-30,000 \mathrm{eV}, z=1-92$, At. Data Nucl. Data Tables 54, 181 (1993).

[8] S. Valencia, A. Gaupp, W. Gudat, H.-Ch. Mertins, P. M. Oppeneer, D. Abramsohn, and C. M. Schneider, Faraday Rotation Spectra at Shallow Core Levels: $3 p$ Edges of Fe, Co, and Ni, New J. Phys. 8, 254 (2006).

[9] J. Zak, E. R. Moog, C. Liu, and S. D. Bader, Magnetooptics of Multilayers with Arbitrary Magnetization Directions, Phys. Rev. B 43, 6423 (1991).

[10] C. Dehesa-Martínez, L. Blanco-Gutierrez, M. Vélez, J. Díaz, L.M. Alvarez-Prado, and J.M. Alameda, Magneto-optical Transverse Kerr Effect in Multilayers, Phys. Rev. B 64, 024417 (2001).

[11] A. Kleibert, V. Senz, J. Bansmann, and P. M. Oppeneer, Thickness Dependence and Magnetocrystalline Anisotropy of the X-Ray Transverse Magneto-optical Kerr Effect at the Co $2 p$ Edges of Ultrathin Co Films on W(110), Phys. Rev. B 72, 144404 (2005).

[12] C. Wang, T. Araki, and H. Ade, Soft X-Ray Resonant Reflectivity of Low-Z Material Thin Films, Appl. Phys. Lett. 87, 214109 (2005). 